\title{
Studies in Molecular Weight Determination of Cottonseed and Melon Seed Oils Based Biopolymers
}

\author{
Ibanga 0. Isaac*, Edet W. Nsi \\ Department of Chemistry, Akwa Ibom State University, Uyo, Nigeria \\ Email: ${ }^{*}$ ibekaunderscoreisaac@yahoo.com
}

Received 29 October 2014; revised 26 November 2014; accepted 3 December 2014

Copyright (C) 2015 by authors and Scientific Research Publishing Inc.

This work is licensed under the Creative Commons Attribution International License (CC BY). http://creativecommons.org/licenses/by/4.0/

(c) (i) Open Access

\section{Abstract}

Six grades of biopolymers formulated to have oil content of $40 \%\left(M_{1}\right), 50 \%\left(M_{2}\right)$, and $60 \%\left(M_{3}\right)$ melon seed oil (MESO) and $40 \%\left(C_{1}\right), 50 \%\left(C_{2}\right)$, and $60 \%\left(C_{3}\right)$ cottonseed oil (COSO) respectively, were prepared with phthalic anhydride, and glycerol using alcoholysis-polycondensation process. The extend of polycondensation was monitored by determining the acid value of aliquots of the reaction mixture at various intervals of time. Molecular weight averages and polydispersity index (PDI) of the finished alkyds were determined by Rast method and end-group analysis. Molecular weight averages and PDI vary with differences in oil length of the alkyds, with samples $M_{2}$ and $C_{2}$ respectively exhibiting the highest PDI. Molecular weight average obtained from end-group analysis and those determined by Rast method in brackets are $1338.92(597.00), 982.33$ (696.25), 1316.09 (754.03), and 1160.57 (448.13), 765.96 (583.57), 1049.92 (696.25) for samples $M_{1}, M_{2}, M_{3}$ and $C_{1}, C_{2}, C_{3}$ respectively. Number molecular weight averages calculated from end-group analysis are larger than those obtained by Rast method for both MESO and COSO alkyds and seem to grossly overestimate their molecular weights. The mode of variation of these properties indicates that the synthesis of MESO and COSO alkyds are complex. Correlation of PDI with the quality of the finished alkyds shows that the higher the PDI value the better the quality of the alkyd. Performance properties such as rate of drying, film hardness and resistance to chemicals were optimum at $\mathbf{5 0 \%}$ oil length for both triglyceride oil alkyds.

\section{Keywords}

Biopolymers, End-Group Analysis, Molecular Weight Averages, Polydispersity Index, Rast Method

\footnotetext{
${ }^{*}$ Corresponding author.
} 


\section{Introduction}

Alkyd resins have been identified as one of the biopolymers synthesised from triglyceride oils [1]. It is a key ingredient of all surface-coating products like paints, primer, adhesives, printing ink [2] and varnishes. They are tough resinous materials prepared via an esterification reaction among polybasic acids, polyols and monoacids (commonly fatty acids from vegetable oils or fats) [3].

Alkyd resins have been recognized as complex systems because of the variety of ingredients used in their preparation and the possibility of formation of three-dimensional network [4] [5]. Alkyd resin usually consists of mixtures of low, medium and high molecular weight species. Hence, its properties depend on the average molecular weight and molecular weight distribution. It is well known that the end-use properties of alkyd resin as coatings become optimum at a region along the extent of reaction co-ordinate when appreciable larger molecules begin to form, i.e., cross-linking of molecular chains [5] [6]. Thus, the physical, chemical and mechanical properties of alkyd resins like other polymers are dependent on their relative sizes or molecular weight [5] [7] [8].

Generally, properties of polymers are determined by the molecular properties-molecular structure, polarity and flexibility of the polymer chains. For instance, rate of oxidation of film deposited, and its toughness and resistance to degradation, have been found to be related to the molecular weight of the alkyd [7] [9]. Similarly, the stability of alkyds on storage and their solubility in conventional hydrocarbon solvents, for example, white spirit, commonly employed in modifying their viscosity, are both dependent on their average molecular weights [8]. Therefore, knowledge of the average molecular weight and molecular weight distribution of the finished alkyds is of utmost importance in their practical application [8] and as a means of evaluating their performance as binders in surface coatings.

However, in processing alkyds for optimum coating performance, it is required that the molecular weights are not too large; otherwise, the alkyd may convert into an intractable gel and become out of control or too low such that its coating performance is impaired [8]. Thus, alkyd resins of appreciable molecular sizes that would ensure trouble-free processing, exhibit stability on storage and perform well during application are desirable for the alkyd chemists and technologists.

In our previous studies [10] [11], it has been discovered that melon seed and cottonseed oils are potential raw material for substituting imported drying oils for use in the Nigerian surface coating industry. Hence, the need for further research involving the determination of molecular weight of these products uses different methods in order to ascertain the method suitable for routine analysis of the alkyds.

The use of cryoscopic or Rast method for determination of the weight-average molecular weight, $\bar{M}_{w}$, number-average molecular weight, $\bar{M}_{n}$, and polydispersity of alkyd resins have been reported [4] [8] [12]-[14]. Absolute methods of molecular weight determination such as light scattering and osmometry require sophisticated and expensive equipment, often; experiments take long time to complete. Many industries cannot afford such sophisticated equipment and lack personnel to handle it. Thus, such methods are unsuitable for routine analysis required in the surface coating industry, since empirical rather than absolute molecular weights are needed. This research is therefore aimed at comparing the average molecular weights of cottonseed and melon seed oils alkyd resins obtained by end-group analysis and Rast method, in order to ascertain the method suitable for routine analysis of molecular weights of these alkyd resins.

\section{Materials and Methods}

\subsection{Materials}

Triglyceride oils used for alkyd resins preparation were locally sourced. COSO was purchased at Sabongari market, Kano, while melon seeds were purchased at Akpan Andem market, Uyo, processed and the oil soxhlet extracted using the method described in our previous work [11]. Technical grade phthalic anhydride, glycerol, xylene and lead (II) oxide were obtained from commercial sources and used without further purification in the preparation of COSO and MESO alkyd resins. A micro-analytical reagent (MAR) camphor obtained from commercial source was used in the determination of melting points of unfractionated alkyd samples.

\subsection{Preparation of Alkyd Resins}

Six grades of alkyd resins formulated to have oil content of $40 \%\left(M_{1}\right)$, 50\% $\left(M_{2}\right)$, and $60 \%\left(M_{3}\right)$ MESO and $40 \%$ $\left(C_{1}\right), 50 \%\left(C_{2}\right)$, and $60 \%\left(C_{3}\right)$ COSO respectively, which were prepared with phthalic anhydride, glycerol and 
xylene in the previous study [10], were used in this experiment. Aliquots of the reaction mixture were withdrawn at 20 min intervals and the acid value determined by titrimetric method [15]. The recipe for the formulation of short, medium and long oil alkyds of MESO and COSO respectively reported elsewhere [16].

\subsection{Determination of Molecular Weights of Alkyd Samples}

Rast method and end-group analysis were employed in the determination of the average molecular weights of the alkyd samples.

\subsection{Cryoscopy (Rast Method)}

Number-average molecular weights $\left(\bar{M}_{\text {det }}\right)$ of the finished alkyds were determined by the cryoscopic method employing the freezing-point depression of camphor [14]. Alkyd sample (0.05 g) was weighed into a test tube, then, $0.5 \mathrm{~g}$ of pure camphor (MAR) was weighed into the test tube. The test tube was stoppered loosely with a cotton wool and the content was melted by placing it in an oil bath, previously heated to about $140^{\circ} \mathrm{C}$, for about $1 \mathrm{~min}$. It was allowed to cool down and the content was transferred to a clean watch glass and powdered. Some of the powder was introduced into a thin capillary tube of which one end was carefully sealed in a flame and its melting point determined. The $\left(\bar{M}_{\text {det }}\right)$ was computed from the expression in Equation (1).

$$
\bar{M}_{\text {det }}=\frac{k \times y \times 1000}{\left(T_{2}-T_{1}\right) \times Z}
$$

where $y$ is the weight (g) of alkyd sample, $Z$ is the weight (g) of pure camphor, $T_{1}$ is the melting point of the mixture of camphor and alkyd, $T_{2}$ is the melting point of pure camphor and $k$ is the molecular depression constant of camphor and equals 39.7 [14].

\subsection{End-Group Analysis}

The extent of the reaction $\left(P_{n}\right)$, and number-average degree of polymerization $\left(\bar{X}_{n}\right)$ calculated in our previous work [1] were used in the determination of weight-average molecular weight $\left(\bar{M}_{w}\right)$, and number-average molecular weight $\left(\bar{M}_{n}\right)$, as well as PDI based on Equations (2), (3) and (5) respectively.

$$
\bar{M}_{w}=\frac{w}{\left(k-P_{n}\right) e_{A}}
$$

where $w$ is the total weight of all the ingredients charged, $k$, the ratio of the total moles of all ingredients charged to total equivalents of the acid $\left(M_{o} / e_{A}\right)$; and $e_{A}$, the total equivalent of the acid. These parameters were calculated in our previous studies [16].

$$
\bar{M}_{n}=\frac{M_{o}}{\left(1-P_{n}\right)}
$$

where $M_{o}$ is the average molecular weight of the repeating unit of the alkyd and it is expressed as follows:

$$
M_{o}=\frac{Q(\mathrm{MW})_{1}+P(\mathrm{MW})_{2}+G(\mathrm{MW})_{3}}{Q+P+G}
$$

where $Q, P$ and $G$ are respectively the number of moles of the oil, diacid and glycerol used in the formulation [16] and $\mathrm{MW}_{1}=265.95, \mathrm{MW}_{2}=132.00$, and $\mathrm{MW}_{3}=89.60$ are the approximate molecular weights of oil, diacid and glycerol, respectively, corrected for the possible loss of end groups [5] [7] [8].

$$
\bar{M}_{w} / \bar{M}_{n}=\text { PDI }
$$

\section{Results and Discussion}

\section{Molecular Weight Characterization of MESO and COSO Alkyd Resins}

It is observed from Figure 1 and Table 1 that during the early stages (up to 60 min) of MESO alkyd synthesis, there seems to be no substantial increase in the molecular sizes of the reaction mixture. However, an appreciable 


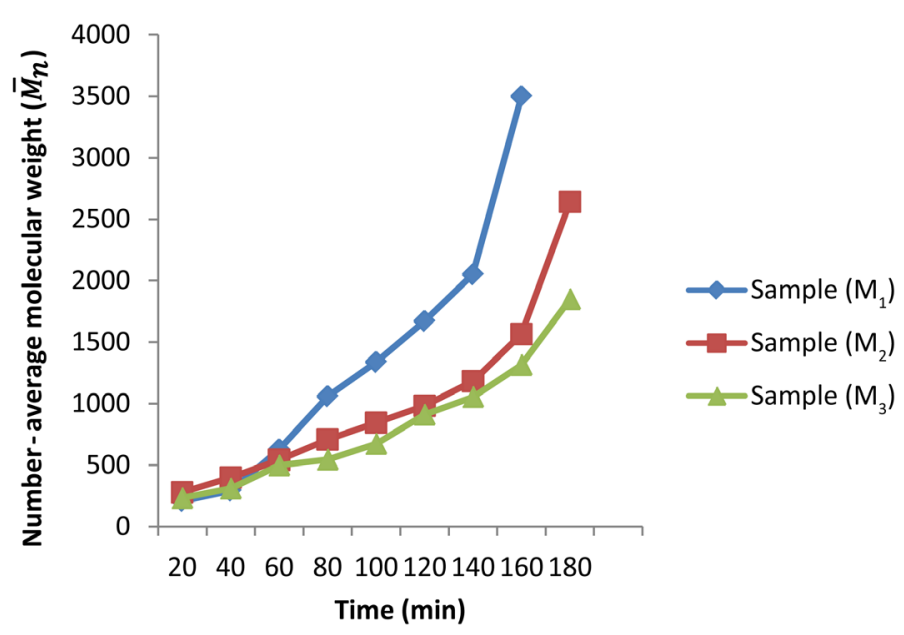

Figure 1. Plot of number-average molecular weight of melon seed oil alkyd sample $M_{1}, M_{2}$ and $M_{3}$ against reaction time.

Table 1. Weight-average molecular weight $\left(\bar{M}_{w}\right)$ of melon seed and cottonseed oil-modified alkyd resins at various stages of reaction.

\begin{tabular}{ccccccc}
\hline Time & Sample $\left(M_{1}\right)$ & Sample $\left(M_{2}\right)$ & Sample $\left(M_{3}\right)$ & Sample $\left(C_{1}\right)$ & Sample $\left(C_{2}\right)$ & Sample $\left(C_{3}\right)$ \\
\hline 20 & 232.38 & 335.27 & 252.39 & 197.350 & 235.63 & 251.47 \\
40 & 340.37 & 539.76 & 344.94 & 250.920 & 320.72 & 347.71 \\
60 & 891.80 & 872.093 & 591.90 & 442.217 & 670.54 & 586.63 \\
80 & 2112.08 & 1434.03 & 659.30 & 1148.545 & 826.45 & 644.88 \\
100 & 3597.12 & 2215.66 & 854.96 & 1872.659 & 1390.18 & 860.09 \\
120 & 7727.98 & 3588.52 & 1275.92 & 2551.020 & 1716.25 & 1232.54 \\
140 & 51724.14 & 10416.67 & 1576.27 & 9554.140 & 5474.45 & 1564.13 \\
\hline
\end{tabular}

$M_{1}-40 \%$ MESO alkyd sample; $M_{2}-50 \%$ MESO alkyd sample; $M_{3}-60 \%$ MESO alkyd sample; $C_{1}-40 \%$ COSO alkyd sample; $C_{2}-50 \%$ COSO alkyd sample; $C_{3}-60 \%$ COSO alkyd sample.

increase was observed as the reaction progressed. Similar observation was made for COSO alkyd samples $\left(C_{1}\right)$ $\left(C_{3}\right)$, such that during the early stages of reaction (up to $80 \mathrm{~min}$ ), no substantial increase in the molecular weights were observed (Figure 2 and Table 1).

However, an appreciable increase was observed, especially for sample $\left(M_{1}\right)$ after about 120 min followed by sample $\left(M_{2}\right)$ and finally $M_{3}$ having the least values for MESO alkyd samples. Similarly, the molecular sizes of COSO alkyd reaction mixture increases from $C_{1}$ through $C_{2}$ with $C_{3}$ having the least value after 180 min of reaction (Figure 2). This observation may be because the two oils are semi-drying oil [10] [11]. Further more, the shapes of these plots depict the complexity in the changes in the molecular sizes of the reaction mixture. The low molecular weight of the alkyds observed at the beginning of the reaction was attributed to the low rate of polymerization. Increase in the polymerization rate resulted in the corresponding increase in molecular weight. This trend continued until the gelation point, at which structural changes began to occur in the polymer size of the molecules in solution. This observation is in agreement with literature reports for castor oil and rubber seed oil alkyds [5] [8] [17]. It is also evident from Figure 1 and Figure 2 and Table 1 that the number-average molecular weight as well as weight-average molecular weight of the in-process samples decreased with an increase in the oil lengths as also reported in literature for rubber seed oil and castor oil alkyd resins [8] [17]. Nagata [6] suggested that an increase in oil length increases the amount of fatty acid available for reaction, which in turn results in a higher chance of termination of chain growth, resulting in lower molecular weight polymers. The above statement is applicable if fatty acid is used. On the other hand, when triglyceride oil is used as in this 
research, the termination might be due to the possibility of the presence of traces of diglycerides in the reaction mixture, if the triglycerides were not completely converted to monoglycerides during the alcoholysis stage of the reaction. The presence of diglycerides can terminates the polymerization and hence low chain length. The trend in the variations of molecular weights of MESO alkyds were as follows: $40 \%$ crude melon seed oil alkyd sample $\left(M_{1}\right)$ greater than $50 \%$ crude melon seed oil alkyd sample $\left(M_{2}\right)$ and $60 \%$ crude melon seed oil alkyd sample $\left(M_{3}\right)$. Those of COSO alkyds were similar to its MESO counterparts: $C_{1}$ greater than $C_{2}$ and $C_{3}$.

The weight—average molecular weight, $\bar{M}_{w}$, the number—average molecular weight $\bar{M}_{n}$ and polydispersity of MESO and COSO alkyd resins calculated at the region where deviation from linearity was observed in Figure 1 and Figure 2 as well as average molecular weight determined using cryoscopic method, $\bar{M}_{\text {det }}$, are presented in Table 2. On the other hand, Figure 3 and Figure 4 compare the $\bar{M}_{\text {det }}$ and $\bar{M}_{n}$ of MESO and COSO alkyd resins respectively.

From Table 2 and Figure 3, it is observed that $\bar{M}_{w}$, decreases from 3597.12 for sample $M_{1}, 3588.52$ for sample $M_{2}$ and 1576.27 for alkyd sample $M_{3}$. $\bar{M}_{n}$ values range from 982.33 for sample $M_{2}, 1316.09$ for sample $M_{3}$ and 1338.92 for sample $M_{1}$. Values of $\bar{M}_{\text {det }}$ on the other hand, increases from 597.00 for sample $M_{1}$, 696.25 for sample $M_{2}$ and 754.03 for sample $M_{3}$.

The $\bar{M}_{n}$ obtained at the region where there was deviation from linearity ranged from 765.96 for alkyd sample $C_{2}, 1049.92$ for alkyd sample $C_{3}$ and 1160.57 for cottonseed oil alkyd sample $C_{1}$. Also, values of $\bar{M}_{w}$ decreases from 2551.02 for sample $C_{1}, 1716.25$ for sample $C_{2}$ and 1564.13 for sample $C_{3}$. On the other hand,

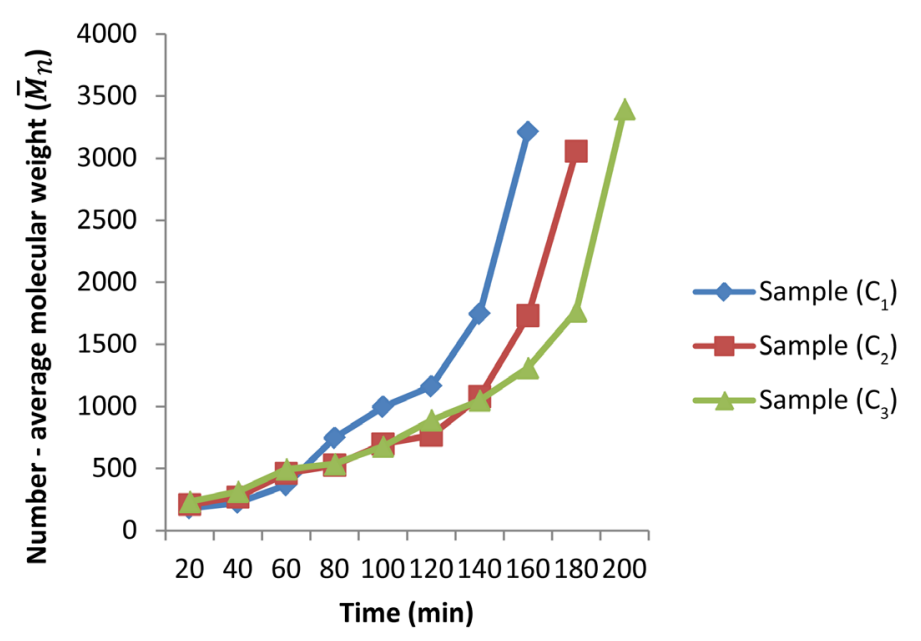

Figure 2. Plot of number-average molecular weight of cottonseed oil alkyd sample $C_{1}, C_{2}$ and $C_{3}$ against reaction time.

Table 2. Molecular weight averages of MESO and COSO alkyds obtained by cryoscopy $\left(\bar{M}_{\text {det }}\right)$, and those obtained by end-group analysis (at the point of deviation from linearity from Figure 1, Figure 2) ( $\bar{M}_{w}$ and $\bar{M}_{n}$ ) and polydispersity index (PDI).

\begin{tabular}{ccccc}
\hline \multirow{2}{*}{ Alkyd Sample } & \multicolumn{3}{c}{ Average molecular weight } & PDI \\
\cline { 2 - 4 } & $\bar{M}_{\text {det }}$ & $\bar{M}_{\mathrm{w}}$ & $\bar{M}_{n}$ & $\left(\bar{M}_{\mathrm{w}} / \bar{M}_{n}\right)$ \\
\hline$M_{1}-40 \%$ MESO alkyd sample & 597.00 & 3597.12 & 1338.92 & 2.69 \\
$M_{2}-50 \%$ MESO alkyd sample & 696.25 & 3588.52 & 982.33 & 3.65 \\
$M_{3}-60 \%$ MESO alkyd sample & 754.03 & 1576.27 & 1316.09 & 1.19 \\
$C_{1}-40 \%$ COSO alkyd sample & 448.13 & 2551.02 & 1160.57 & 2.19 \\
$C_{2}-50 \%$ COSO alkyd sample & 583.57 & 1716.25 & 765.96 & 2.24 \\
$C_{3}-60 \%$ COSO alkyd sample & 696.25 & 1564.13 & 1049.92 & 1.49 \\
\hline
\end{tabular}




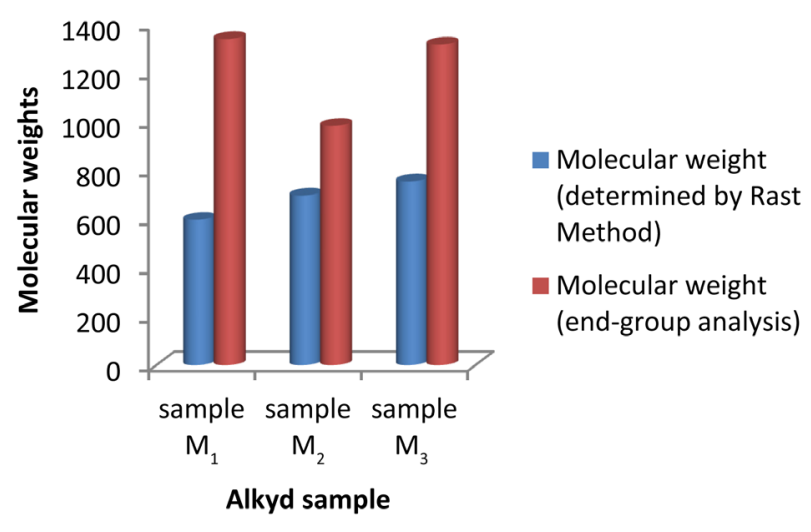

Figure 3. Molecular weights $\left(\bar{M}_{\text {det }}\right.$ and $\bar{M}_{n}$ ) for melon seed oil alkyd sample $\left(M_{1}\right),\left(M_{2}\right)$ and $\left(M_{3}\right)$.

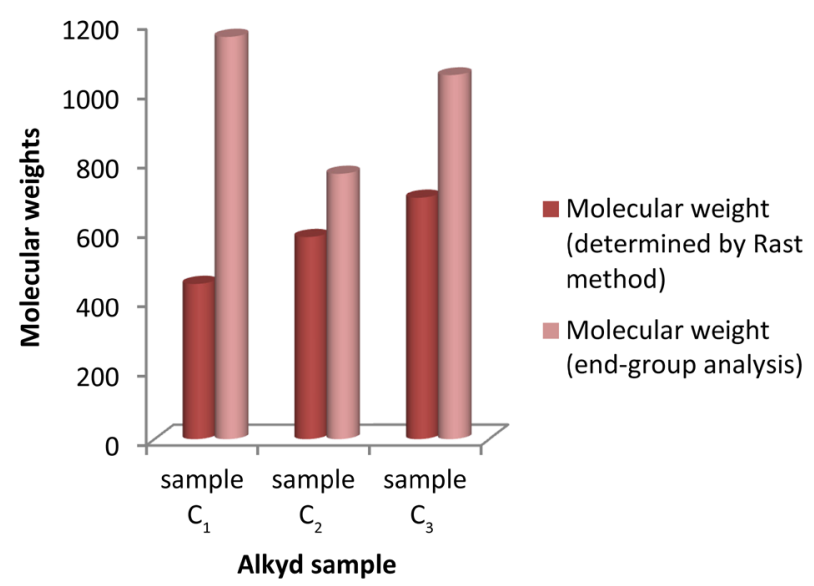

Figure 4. Molecular weights $\left(\bar{M}_{\text {det }}\right.$ and $\left.\bar{M}_{n}\right)$ for cottonseed oil alkyd samples $\left(C_{1}\right),\left(C_{2}\right)$ and $\left(C_{3}\right)$.

$\bar{M}_{\text {det }}$ value for cottonseed oil alkyd samples increases from 448.13 for sample $C_{1}, 583.57$ for sample $C_{2}$ and 696.25 for sample $C_{3}$ (Table 2 and Figure 4). These results is in collaboration with those of crude melon seed oil alkyds counterpart above and are comparable with those reported in the literature for rubber seed oil alkyds resins [4]. It differs from those reported by [8] in which the $\bar{M}_{n}, \bar{M}_{w}$ and PDI in parentheses for short, medium and long oil alkyd samples of rubber seed oil obtained by gel permeation chromatography were 3234, 6186 (1.9); 1379, 2147 (1.56); and 3304, 8406 (2.56) respectively. The medium oil had the least PDI value, while in this research; the medium oil had the highest PDI value. This could be attributed to the difference in method used for obtaining $\bar{M}_{n}$ and $\bar{M}_{w}$ values, which in this case is end-group analysis. The PDI values as observed in Table 3 vary with reaction time with short and medium oil alkyd of both MESO and COSO alkyd samples being higher than that of long oil alkyds of the two oil samples. Hence, the PDI obtained by end-group analysis increases with increase degree of polymerization. The unusual high value of PDI of 23.63 for $M_{1}$ compared to it $C_{1}$ counterpart of 5.48 (Table 3), could be due the fact that crude and not refined MESO was used for preparation alkyd sample $M_{1}$, while refined COSO was used for preparation of alkyd sample $C_{1}$.

These molecular weight averages and PDI show that the reaction mixture is constituted of low molecular weight species. This could be due to the possibility of the presence of diglycerides, which terminates the polymerization, and hence low chain length. The PDI clearly indicate that the size distribution is broad. However, 50\% MESO alkyd sample $M_{1}$ and 50\% COSO alkyd sample $C_{1}$ with the highest PDI value of 3.652 .24 respectively can be considered as having the broadest molecular weight distribution.

The results on Table 2 as well as Figure 3 and Figure 4 also show that $\bar{M}_{n}$ calculated from end-group analysis are larger than $\bar{M}_{\text {det }}$ determined from Rast method for both melon seed oil and cottonseed oil alkyds. 
Table 3. Polydispersity index of MESO and COSO alkyd samples.

\begin{tabular}{ccccccc}
\hline Time & Sample $\left(M_{1}\right)$ & Sample $\left(M_{2}\right)$ & Sample $\left(M_{3}\right)$ & Sample $\left(C_{1}\right)$ & Sample $\left(C_{2}\right)$ & Sample $\left(C_{3}\right)$ \\
\hline 20 & 1.11 & 1.20 & 1.08 & 1.10 & 1.12 & 1.08 \\
40 & 1.16 & 1.35 & 1.12 & 1.12 & 1.19 & 1.11 \\
60 & 1.42 & 1.60 & 1.19 & 1.22 & 1.45 & 1.19 \\
80 & 1.99 & 2.03 & 1.21 & 1.54 & 1.57 & 1.20 \\
100 & 2.69 & 2.62 & 1.27 & 1.88 & 2.24 & 1.27 \\
120 & 4.62 & 3.65 & 1.40 & 2.20 & 5.08 & 1.39 \\
140 & 23.63 & 8.81 & 1.49 & 5.48 & & 1.49 \\
\hline
\end{tabular}

The discrepancy between $\bar{M}_{\text {det }}$ and $\bar{M}_{n}$ may be attributed to the assumptions made in the end-group analysis to the effect that the reactivity of the polymer chains is independent of its size, that the functional groups of the same kind are equally reactive, that intramolecular reactions are absent, and that only interesterification reaction occurs during polyesterification [4] [8] [13]. Actually, occurrence of intramolecular condensation or cyclization leading to ring formation during polyesterification has been reported [12]. Since an intramolecular reaction could occur during alkyd preparation leading to a decrease in concentration of carboxyl groups without a corresponding increase in chain length, there will be an increase in extent of reaction and consequently average degree of polymerization calculated thereof. It is not surprising therefore, that in the present study, $\bar{M}_{n}$, calculated from end - group analysis is larger than those determined by Rast method $\left(\bar{M}_{\text {det }}\right)$. Hence, end-group analysis seems to grossly overestimate the $\bar{M}_{n}$ of the alkyd resins. Indeed, similar discrepancies between $\bar{M}_{\text {det }}$ and $\bar{M}_{n}$ have been reported [5] [8] [18].

Previous studies on molecular weight determination of some alkyds indicated that they are constituted of species of relatively low molecular weights of approximately 500 [12] [18], although molecular weights of between 1170 and 1250 have been reported [6]. It is observed that both $\bar{M}_{\text {det }}$ and $\bar{M}_{n}$ for all the alkyd samples studied fall within the range reported by Aigbodion and Pillai [8] and Flory [12].

Correlation of the performance characteristics of the melon seed and cottonseed oils alkyds obtained in our previous studies [10] [11] [19] with their number-average molecular weights and PDI shows that the lower the polydispersity index of the alkyds are the less is their performance as binders. Thus, $50 \%$ oil length alkyds $\left(M_{2}\right.$ and $C_{2}$ ), which has the highest PDI, exhibited the best characteristics such as excellent adhesion, good hardness, fast drying time and resistance to chemicals followed by $40 \%$ oil length alkyds ( $M_{1}$ and $C_{1}$ ). $60 \%$ oil length alkyds $\left(M_{3}\right.$ and $\left.C_{3}\right)$ with the least PDI had the least performance characteristics.

\section{Conclusion}

In conclusion, it can be inferred from these results that reactions leading to the formation of melon seed and cottonseed oils alkyd resins are complex. The PDI of MESO and COSO alkyd resins is an important parameter, which determines their performance as binders in surface coating products. Average molecular weights determined by Rast method increased with increase in oil length of the alkyds and were less than those obtained from end-group analysis. It is found in this study that Rast method is more reasonable than end-group analysis for routine determination of molecular weights of alkyd resins.

\section{References}

[1] Isaac, I.O. and Ekpa, O.D. (2014) Comparative Study on the Kinetics of the Preparation of Melon Seed and Cottonseed Oils Based Biopolymers. American Journal of Polymer Science, 4, 7-15.

[2] Uzoh, C.F., Onukwuli, O.D., Odera, R.S. and Ofochebe, S. (2013) Optimization of Polyesterification Process for Production of Palm Oil Modified Alkyd Resin Using Response Surface Methodology. Journal of Environmental Chemical Engineering, 1, 777-785. http://dx.doi.org/10.1016/j.jece.2013.07.021

[3] Ling, J.S., Mohammed, I.A., Ghazali, A. and Khairuddean, M. (2014) Novel Poly (Alkyd-Urethane)s from Vegetable Oils: Synthesis and Properties. Industrial Crops and Products, 52, 74-84. http://dx.doi.org/10.1016/j.indcrop.2013.10.002 
[4] Aigbodion, A.I. and Okieimen, F.E. (1996) Kinetics of the Preparation of Rubber Seed Oil Alkyds. European Polymer Journal, 32, 1105-1108. http://dx.doi.org/10.1016/0014-3057(96)00053-5

[5] Okieimen, F.E. and Aigbodion, A.I. (1997) Studies in Molecular Weight Determination of Rubber Seed Oil Alkyds. Industrial Crops and Products, 6, 155-161. http://dx.doi.org/10.1016/S0926-6690(96)00209-9

[6] Nagata, T. (1969) Cooking Schedule of Alkyd Resin Preparation-Part II. Effect of Cooking Schedule on Molecular Weight Distribution of Alkyd Resin. Journal of Applied Polymer Science, 13, 2601-2619. http://dx.doi.org/10.1002/app.1969.070131208

[7] Bobalek, E.G., Moore, E.R., Levy, S.S. and Lee, C.C. (1964) Some Implications of Gel Point Concept on the Chemistry of Alkyd Resins. Journal of Applied Polymer Science, 8, 625-657. http://dx.doi.org/10.1002/app.1964.070080207

[8] Aigbodion, A.I. and Pillai, C.K. (2001) Synthesis and Molecular Weight Characterization of Rubber Seed Oil-Modified Alkyd Resins. Journal of Applied Polymer Science, 79, 2431-2438. http://dx.doi.org/10.1002/1097-4628(20010328)79:13<2431::AID-APP1050>3.0.CO;2-A

[9] Flory, P.J. (1953) Principles of Polymer Chemistry. Cornell, Ithaca, New York.

[10] Isaac, I.O. and Ekpa, O.D. (2013) Fatty Acid Composition of Cottonseed Oil and Its Application in Production and Evaluation of Biopolymers. American Journal of Polymer Science, 3, 13-22.

[11] Ekpa, O.D. and Isaac, I.O. (2013) Fatty Acid Composition of Melon (Colocynthis vulgaris Shrad) Seed Oil and Its Application in Synthesis and Evaluation of Alkyd Resins. IOSR Journal of Applied Chemistry, 4, 30-41. http://dx.doi.org/10.9790/5736-0443041

[12] Flory, P.J. (1941) Molecular Size Distribution 3-Dimensional Polymers I: Gelation. Journal American Chemical Society, 63, 3083-3090. http://dx.doi.org/10.1021/ja01856a061

[13] Stockmayer, W.H. (1952) Molecular Distribution in Condensation Polymerization. Journal of Polymer Science, 9, 6971. http://dx.doi.org/10.1002/pol.1952.120090106

[14] Furniss, B.S., Hannaford, A.J., Rogers, V., Smith, P.W.G. and Tatchel, A.R. (1978) Vogel's Textbook of Practical Organic Chemistry. 4th Edition, ELBS \& Longman, London.

[15] Ekpa, O.D. and Isaac, I.O. (2009) Kinetic Studies on Polyesterification of Unsaturated Oils and Diacids in the Alcoholoysis Process. Research Journal of Applied Science, 4, 125-128.

[16] Isaac, I.O. and Ekpa, O.D. (2013) Comparative Study on Solution Viscosity Properties of Cottonseed and Melon Seed Oils Based Biopolymers. American Journal of Polymer Science, 3, 23-34.

[17] Onukwli, O.D. and Igbokwe, P.K. (2008) Production and Characterization of Castor Oil-Modified Alkyd Resins. Journal of Engineering \& Applied Science, 3, 161-165.

[18] Kienle, R.H., Van der Meulen, P.A. and Petke, F.E. (1939) The Polyhydric Alcohol-Polybasic Acid Reaction III: Further Studies of the Glycerol-Phthalic Acid Reaction. Journal of American Chemical Society, 61, 2258-2268. http://dx.doi.org/10.1021/ja01878a001

[19] Isaac, I.O. and Nsi, E.W. (2013) Influence of Polybasic Acid Type on the Physicochemical and Viscosity Properties of Cottonseed Oil Alkyd Resins. The International Journal of Engineering and Science, 2, 1-14. 
Scientific Research Publishing (SCIRP) is one of the largest Open Access journal publishers. It is currently publishing more than 200 open access, online, peer-reviewed journals covering a wide range of academic disciplines. SCIRP serves the worldwide academic communities and contributes to the progress and application of science with its publication.

Other selected journals from SCIRP are listed as below. Submit your manuscript to us via either submit@scirp.org or Online Submission Portal.
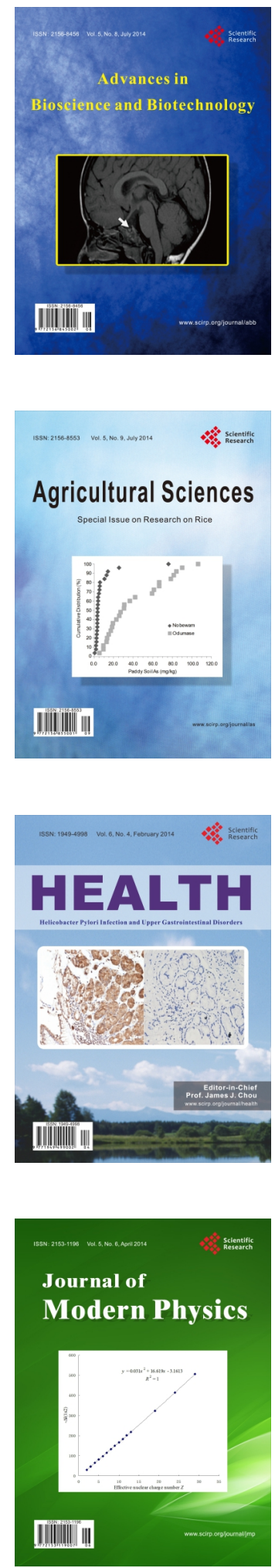
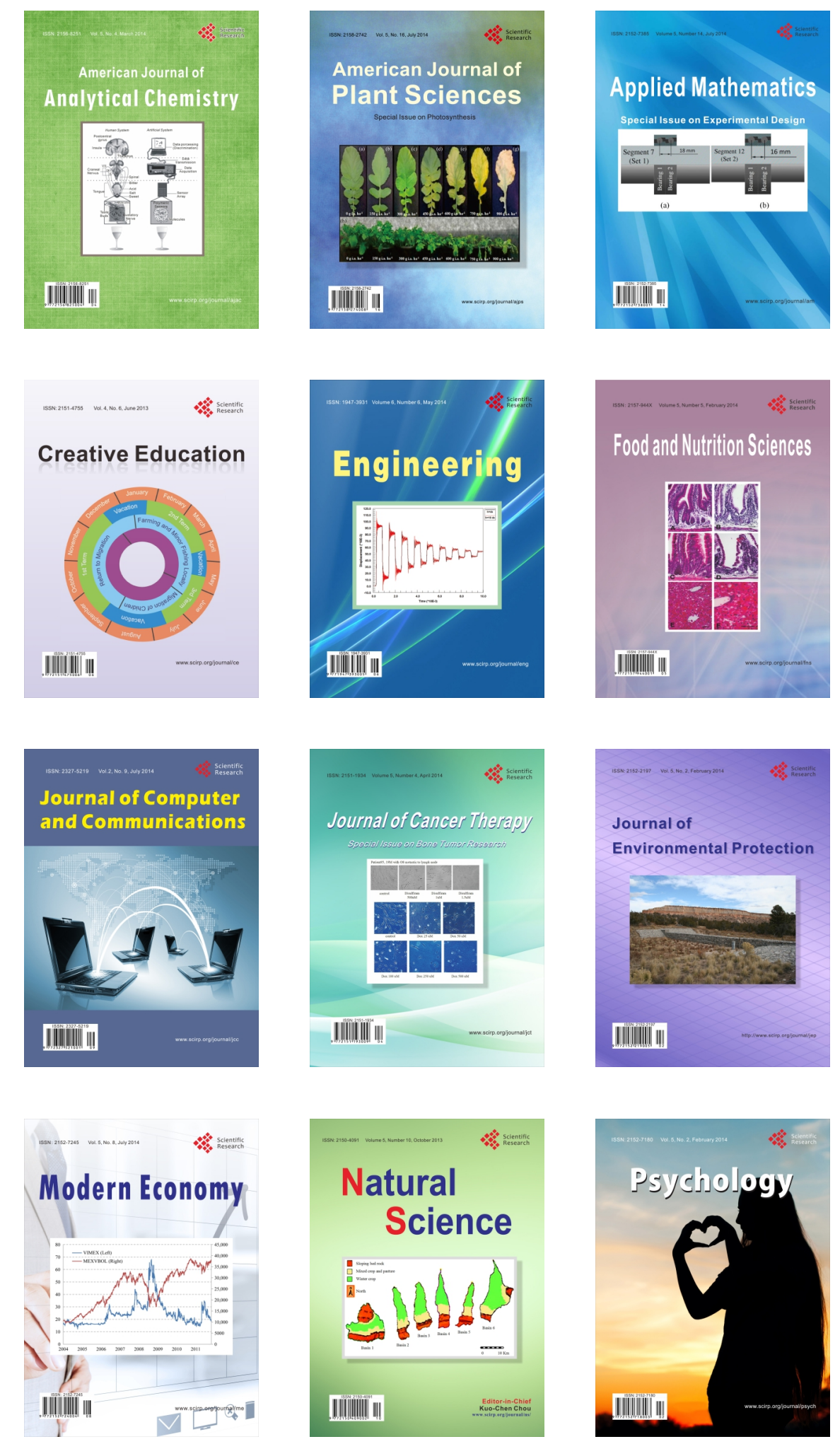